
IP Periodica Polytechnica
Civil Engineering

62(1), pp. 102-111, 2018

https://doi.org/10.3311/PPci.9033

Creative Commons Attribution (i)

RESEARCH ARTICLE

\section{Performance of the CGS six DOF Shaking Table on the Harmonic Signal Reproduction}

\author{
Abdelhalim Airouche ${ }^{1 *}$, Hassan Aknouche ${ }^{1}$, Hakim Bechtoula ${ }^{1}$, \\ Nourredine Mezouer ${ }^{1}$, Abderrahmane Kibboua ${ }^{1}$
}

Received 18 January 2016; Revised 16 February 2017; Accepted 30 May 2017

\begin{abstract}
Shaking table testing continues to play an important role in earthquake engineering research. It has been recognized as a powerful testing method to evaluate structural components and systems under realistic dynamic loads. Although it represents a very attractive experimental procedure, many technical challenges, which require attention and consideration, still remain. High fidelity in signal reproduction is the focus of the work presented in this paper. The main objective of this paper is to investigate the capabilities of adaptive control techniques based on Amplitude Phase Control (APC) and Adaptive Harmonic Cancellation (AHC) on the harmonic signal tracking performance of the shaking table. A series of 232 sinusoidal command waveforms with various frequencies and amplitudes were conducted on the shaking table of the laboratory of the National Earthquake Engineering Applied Research Center (CGS, Algeria). Experimental results are reported and recommendations on the use of these adaptive control techniques are discussed.
\end{abstract}

\section{Keywords}

shaking table, signal reproduction, cross-coupling, amplitude Phase Control, Adaptive Harmonic Cancellation
'National Earthquake Engineering Applied Research Center CGS, Rue Kaddour Rahim Prolongée,

Bp 252 H-Dey Alger, Algeria

"Corresponding author email: airouche@yahoo.fr

\section{Introduction}

Shaking tables are being increasingly used in earthquake engineering research field and remain one of the preferred tools for seismic testing [1]. Their main objective is to replicate a wide range of a desired motion with a high degree of accuracy, in order to generate meaningful and reliable results. However, despite the significant progress achieved in shake table design and control technology during the last decades, number of challenges still exist [2]. These challenges fall into two categories: (i) economical and practical challenges and (ii) technical challenges. In the first category we can cite the problem of the full-scale realistic tests of large structural systems which are constrained by the limited financial resources and capacities of the existing laboratories. The second category includes control issues, consideration of different loading conditions other than dynamic base motion and soil-structure interaction effects in shaking-table testing.

Undoubtedly, the control of shaking table is the key element of the whole system and one of the most difficult technical challenges. In fact, the modern multiple degree-of-freedom shaking table with a resonating and eccentric specimen has the characteristics of a strongly coupled multiple input, multiple output dynamic system [3]. In such a system, servovalves characteristics, oil column resonance, frictions in the system, cross coupling between degrees of freedom, specimen compliance and noise in feedback transducers, are some of the predominant sources of nonlinearities that can adversely affect the accurate control, stability and high fidelity signal reproduction, of the shaking table.

Various control techniques have been developed in order to provide a shaking table with optimum performance and high level of motion control. The earliest methods use conventional linear controller, such as closed-loop PID, deltaP and feed-forward control algorithm $[4,5]$, and three variable control (TVC) $[6,7]$. However, due to the complexity of the problem the need for more sophisticated control systems become necessary. With advances in computing technologies, followed by the breakthrough development of the digital signal processing and realtime operations, methods based on hybrid control strategies 
$[8,9,10]$ and adaptive control techniques $[11,12,13,14,15]$ have been developed. MTS 469D Digital Controller of the shaking table system at the National Earthquake Engineering Applied Research Center, CGS, includes four powerful adaptive control techniques which are: Amplitude Phase Control (APC), Adaptive Harmonic Cancellation (AHC), Adaptive Inverse Control (AIC) and Online Iteration (OLI).

The objective of the present paper concerns the investigation of the capabilities of two of these adaptive control techniques, namely Amplitude Phase Control (APC) and Adaptive Harmonic Cancellation (AHC), on the harmonic signal reproduction. It should be noted that most of the qualification testing, where high-quality of signal tracking is required, use harmonic signal excitations.

However, running sine waves or more generally harmonic signals on shaking table reveal major issues that are: 1) amplitude attenuation, 2) phase shift, and 3) harmonic distortion. In this study these issues were addressed through a comprehensive experimental program performed on the earthquake simulator of the laboratory of CGS. A series of 232 sinusoidal command waveforms with various frequencies and amplitudes were conducted. The test program was divided into two main parts. The first part intends to verify the effectiveness of APC and AHC on the signal reproduction. Whereas, the second part focuses on their effects on reducing the undesirable cross coupling.

Here after, experimental results were reported and recommendations on the use of these adaptive control techniques are discussed.

\section{CGS shaking table system}

The Dynamic testing laboratory at CGS houses a 60tons capacity, six-degree-of-freedom MTS Systems Corporation servo-hydraulic shaking table. It consists of a stiff $6.1 \mathrm{~m}$ by $6.1 \mathrm{~m}$ steel platform weighing 40tons, driven by twelve servohydraulic actuators: four longitudinal, four lateral, and four vertical. The table is capable of simulating earthquake events and other ground vibrations with maximum displacements of $\pm 250 \mathrm{~mm}$ and $\pm 150 \mathrm{~mm}$ in the horizontal directions. The corresponding value of displacement in the vertical direction is \pm 100 $\mathrm{mm}$. Peak velocity of $1.1 \mathrm{~m} / \mathrm{s}$ for each of the two horizontal directions and $1.0 \mathrm{~m} / \mathrm{s}$ in the vertical direction can be achieved. Accelerations of $\pm 1.0 \mathrm{~g}$ for horizontal directions and $\pm 0.8 \mathrm{~g}$ for vertical direction are possible with maximum test specimens of 60 tons. The maximum over turning moment is about 180 ton$\mathrm{m}$. The frequency range is $0-50 \mathrm{~Hz}$.

Hydraulic power that supplies the shake table is provided by a set of five high pressure pumps that can deliver a total flow of 3600 liters per minute at a working pressure of $21 \mathrm{MPa}$. Additional flow capacity can be supplied from accumulators of a total volume of 360 liters for peak flow demands. Fig. 1 shows a global view of the shaking table and its components.

\section{Adaptive control techniques}

As mentioned previously shake tables are highly nonlinear systems. Therefore, high quality waveform reproduction cannot be obtained with conventional controllers which use offline fixed gains, even when the command is a simple sinusoid. Hence, using adaptive control techniques, which enable real time system adaptation to counter parameter changes in system components, are required.

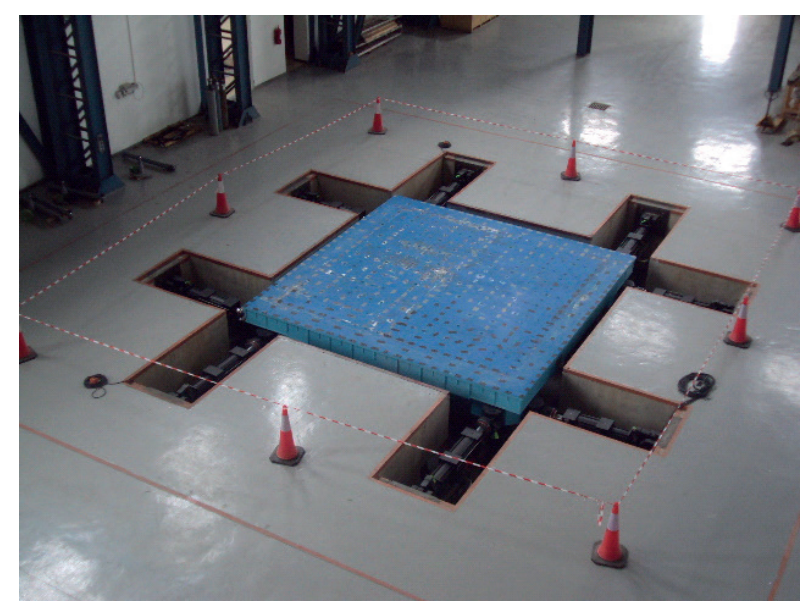

(a)

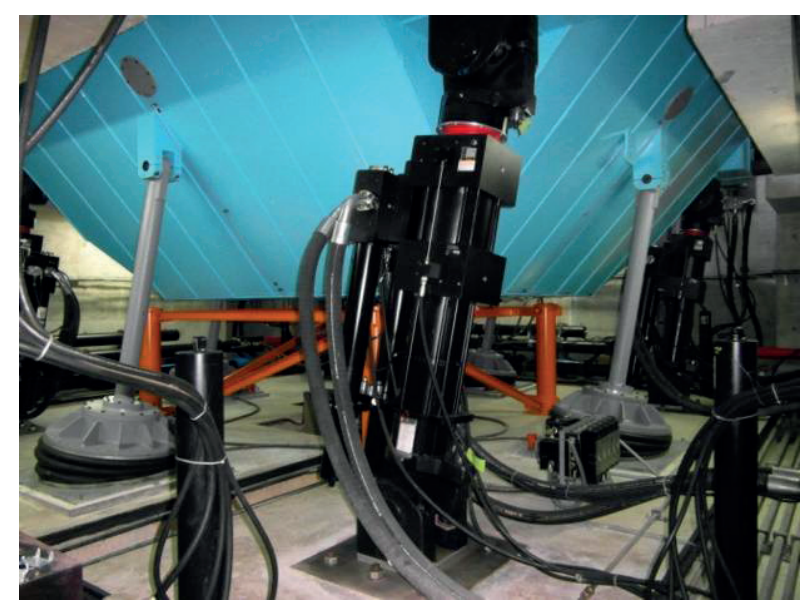

(b)

Fig. 1 View of CGS shaking table: (a) top view and (b) view of the pit area

\subsection{Amplitude Phase Control (APC)}

Fig. 2(a) shows typical discrepancies in amplitude and phase that might exist between command and feedback when the system is reasonably linear. Amplitude/Phase Control (APC), which is optimized to work with sinusoidal command waveforms, is a control technique that fixes these discrepancies. It augments a fixed-gain controller to correct amplitude and phase irregularities, in order to improve the control fidelity. It measures the control system dynamics directly and modifies the control compensation accordingly in real-time [16]. APC is characterized by its fast convergence to the optimum correction because the correction is updated at every point on the sine wave, not just at the peaks. 
(a)

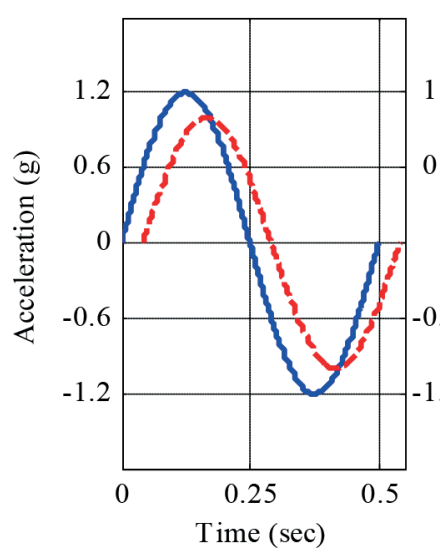

(b)

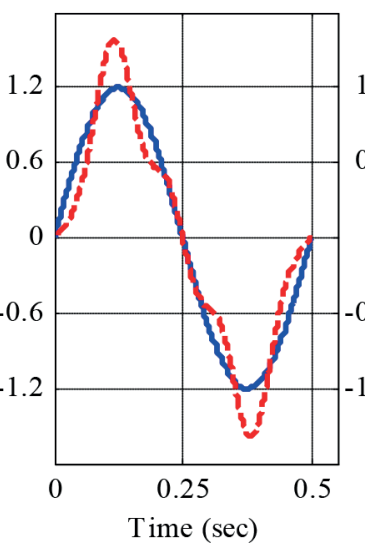

(c)

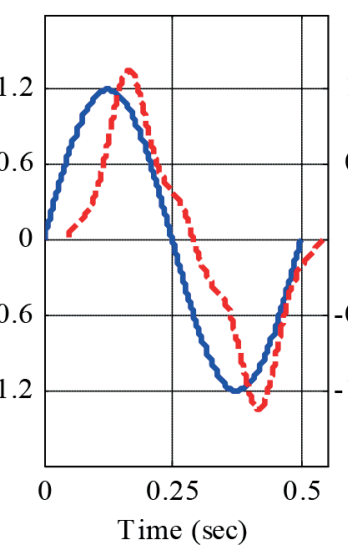

(d)

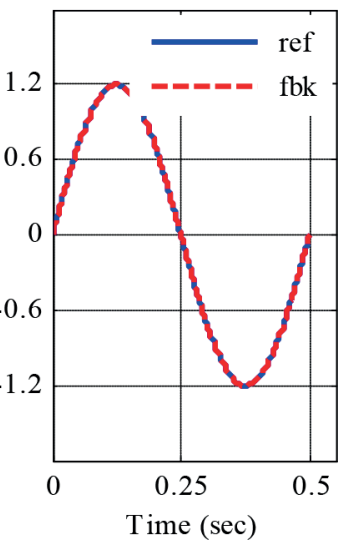

Fig. 2 Type of discrepancies in the shaking table feedbacks, (a) Amplitude and phase errors (b) Spurious harmonics (c) a and b combined (d) Ideal case

\subsection{Adaptive Harmonic Cancellation (AHC)}

A non-linear system like servohydraulic seismic systems will often produce spurious harmonics in its feedback signals even when the command signal is a clean sinusoid; see Fig. 2(b). $\mathrm{AHC}$ is a compensation algorithm designed to remove these unwanted harmonics from a sinusoidal feedback signal [16]. It uses a technique developed in the "adaptive noise control" field that adds harmonics to the controller command signal with the right phase and amplitude to completely cancel the unwanted harmonics at the system output.

Usually, in order to improve the fidelity of the shaking system's response, Fig. 2(c), when performing sine wave testing, both AHC and APC are simultaneously used. APC and AHC complement each other: APC enhances the fundamental frequency component of the system response while AHC cancels the harmonics. Fig. 2(d) shows the ideal case when APC and AHC are correctly used. It can be seen that the feedback is a perfect replicate of the reference.

\section{Testing program}

The testing program is composed of two main parts. The first part deals with the signal reproduction capabilities of APC and $\mathrm{AHC}$ which is discussed in this section, and the second part deals with the cross coupling investigation that it is examined in section 7 .

In order to investigate the harmonic signal tracking performance of the CGS shaking table and the effectiveness of APC and AHC, two groups of tests were performed. The first group, called "uncompensated tests", was conducted without using APC and AHC. The second group, called "compensated tests", was conducted by using APC and AHC. The experimental program for this part was performed by exciting the shaking table (bare table condition) with a comprehensive set of 224 of harmonic acceleration records with frequencies of $2,4,5,6,7,8$, $10,12,15,17,20,25,30,35,40$ and $45 \mathrm{~Hz}$. For each frequency the shaking table was excited with different level of peak amplitudes, namely $0.15,0.20,0.30,0.60,0.75,0.90$ and $1.20 \mathrm{~g}$.

\section{Oil Column}

Oil column resonant frequency was determined to be one of the most significant parameters affecting the dynamic performance of the shaking table system $[18,19]$. As it will be seen in the subsequent sections oil column resonant frequency represents a key factor in interpreting of test results. It is essential, therefore, to determine experimentally this important characteristic, by measuring the open loop shaking table transfer function.

The CGS shaking table's oil column frequencies in the longitudinal, lateral and vertical directions were measured to be 16.40, 17 and $25 \mathrm{~Hz}$, respectively for bare table condition [17]. These frequencies are well within the operating range of the system, [0 50] Hz.

\section{Test results}

Three comparison criteria have been chosen to evaluate the harmonic signal reproduction capability of the CGS shaking table. These include direct comparisons of the acceleration time histories, relative RMS error measure and total harmonic distortion (THD).

\subsection{Acceleration Time Histories Comparison}

In this section qualitative comparison of time history plots between table acceleration reference (ref) and table acceleration feedbacks, (fbk-NC) for uncompensated tests and (fbk-C) for compensated tests, is carried out.

Fig. 3(a) to 3(d) show the table reference and feedbacks (compensated and uncompensated) acceleration time history plots for a set of tests with the same frequency of $2 \mathrm{~Hz}$ and different amplitudes $(0.3,0.6,0.9$ and $1.2 \mathrm{~g})$. These curves bring out the strong nonlinearities that are present in the feedbacks of the uncompensated tests (fbk-NC), which are seriously distorted. It should be emphasized that, the distortion becomes more pronounced as the acceleration amplitude increases. 
(a)

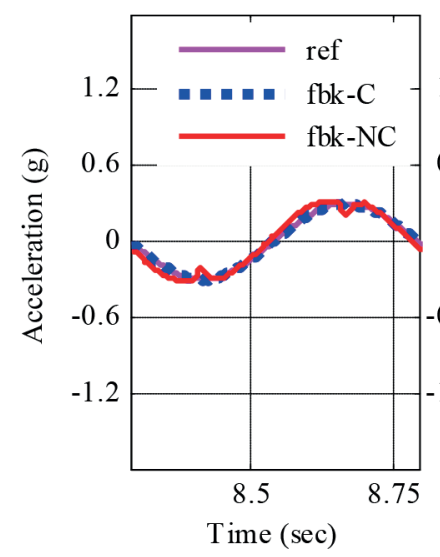

(b)

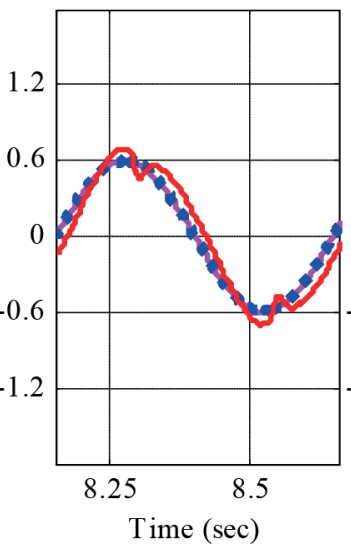

(c)

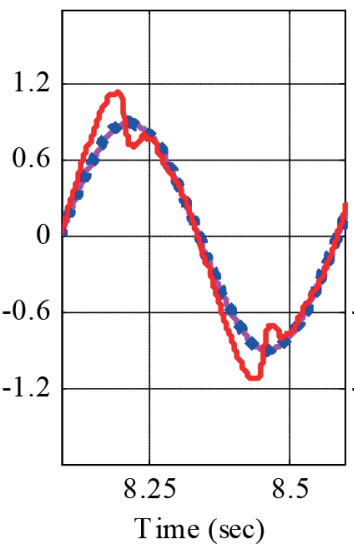

(d)

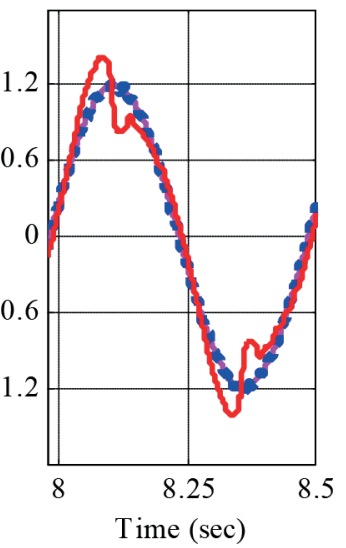

Fig. 3 Shaking table reference (ref) and feedbacks, compensated (fbk-C) and uncompensated (fbk-NC), of harmonic tests at 2 Hz, performed whit peak amplitudes of (a) 0.30 ; (b) 0.60 ; (c) 0.90 and (d) $1.20 \mathrm{~g}$

(a)

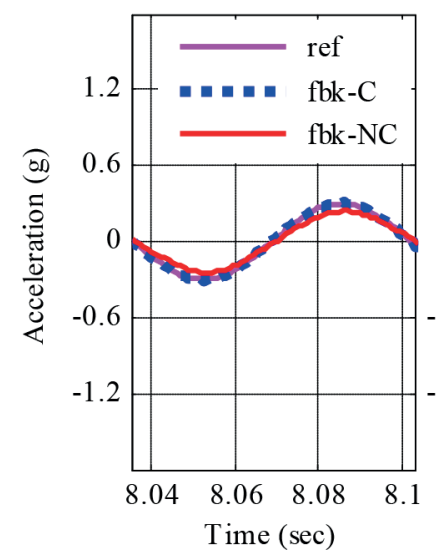

(b)

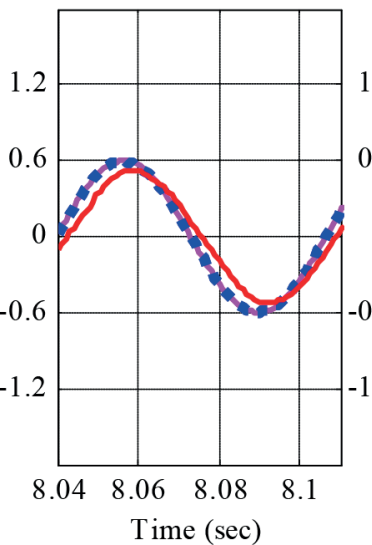

(c)

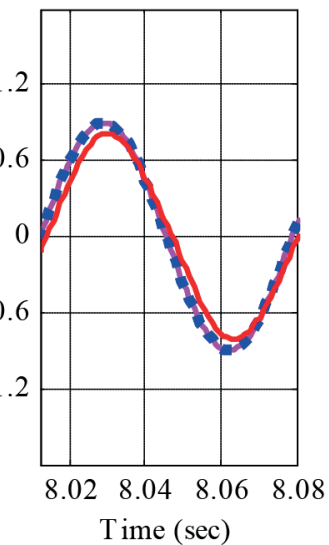

(d)

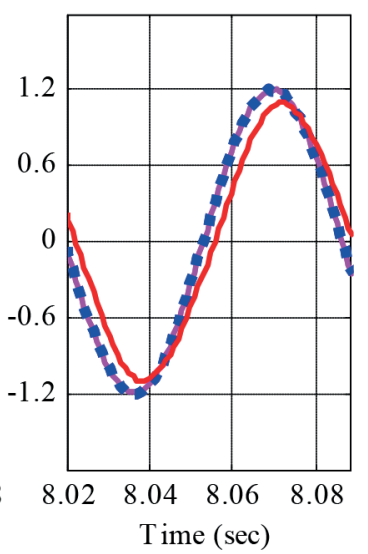

Fig. 4 Shaking table reference (ref) and feedbacks, compensated (fbk-C) and uncompensated (fbk-NC), of harmonic tests at 15 Hz, performed whit peak amplitudes of (a) 0.30 ; (b) 0.60 ; (c) 0.90 and (d) $1.20 \mathrm{~g}$

(a)

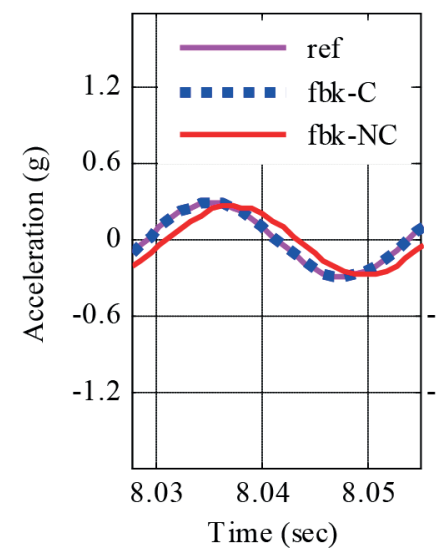

(b)

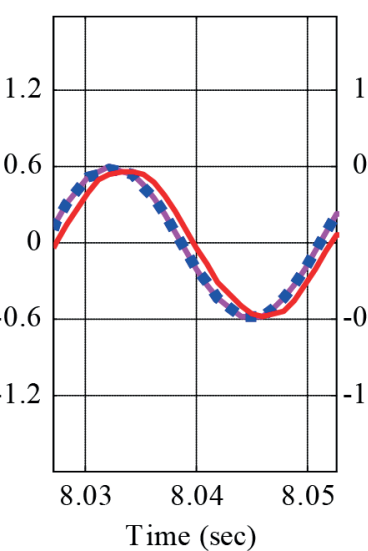

(c)

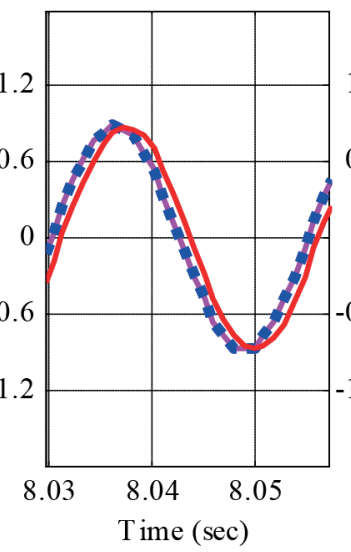

(d)

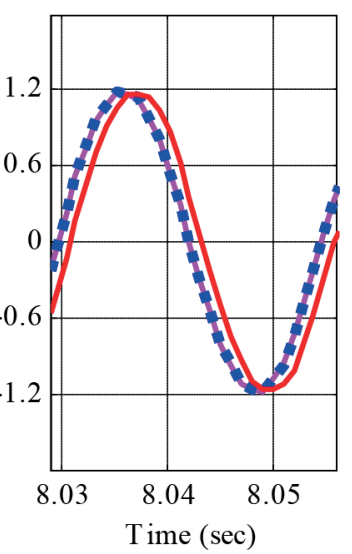

Fig. 5 Shaking table reference (ref) and feedbacks, compensated (fbk-C) and uncompensated (fbk-NC), of harmonic tests at $40 \mathrm{~Hz}$, performed whit peak amplitudes of (a) 0.30 ; (b) 0.60 ; (c) 0.90 and (d) $1.20 \mathrm{~g}$

The same type of plots for the tests at frequencies $15 \mathrm{~Hz}$ and 40Hz is given in Fig. 4(a) to 4(d) and Fig. 5(a) to (d), respectively. What we can note from the curves is the relative linearity in the shaking table feedback for the case of uncompensated tests (fbk-NC), although discrepancies in terms of amplitude and phase are worth noting. Finally, we should make a special note regarding the table acceleration feedback for the case of compensated tests (fbk-C), which is almost a perfect copy of the table reference. This final remark is valid for all compensated tests. 


\subsection{Relative RMS Error Comparison}

In this section relative RMS error measure is used to compare the acceleration reference to the acceleration feedback of the shaking table. The relative RMS error is defined as:

$$
\varepsilon_{r e l}=\frac{\sqrt{(1 / N) \sum_{n=1}^{N}\left(\ddot{x}_{f b k}[n]-\ddot{x}_{r e f}[n]\right)^{2}}}{\sqrt{(1 / N) \sum_{n=1}^{N}\left(\ddot{x}_{r e f}[n]\right)^{2}}} \times 100
$$

Where $\mathrm{N}$ denotes the number of data points within the time window chosen to calculate the error.

In order to get a better understanding and more accurate interpretation of the results, tests are divided into four (04) main groups. The first group corresponds to the harmonic tests with frequencies lower than $1 / 3$ of the oil column $(2,4$, and $5 \mathrm{~Hz}$ ), the second one corresponds to tests with frequencies slightly superior to $1 / 3$ of the oil column frequency $(6,7$ and $8 \mathrm{~Hz}$ ), the third group corresponds to the tests with frequencies close to the oil column $(10,12$ and $15 \mathrm{~Hz})$ and the last one corresponds to high frequency tests $(35,40$ and $45 \mathrm{~Hz})$

Figure 6(a) to 6 (d) shows the relative RMS error curves of the 4 groups obtained for the case of uncompensated tests. It can be seen that the relative RMS error is rather high at low peak amplitudes $(\mathrm{PGA}<0.3 \mathrm{~g})$, with error values of 30 to $40 \%$, then decreases in the intermediate peak amplitudes $(0.3 \mathrm{~g}$ $<\mathrm{PGA}<0.6 \mathrm{~g}$ ). These observations are common to the four groups. However, it should be noted that for the first group, Figure 6(a), the relative RMS errors obtained for the case of large peak amplitudes are as high as the ones of the low peak amplitudes case. This is due mostly to the severe non-linearity in the spool opening of the servovalve caused by pressure switching and flow limits (peak force, peak velocity).

For the second group, Fig. 6(b), the relative RMS error obtained for the case of large peak amplitudes is slightly bigger than those for intermediate peak amplitudes. For the third and forth groups, Fig. 6(c) and 6(d) respectively, the relative RMS error is practically independent of peak amplitudes for values exceeding $0.60 \mathrm{~g}$. Note that small relative RMS errors were achieved for these two groups. (a)

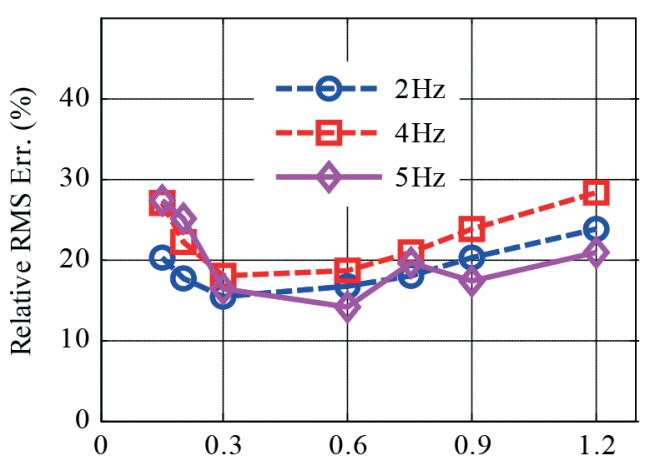

(b)

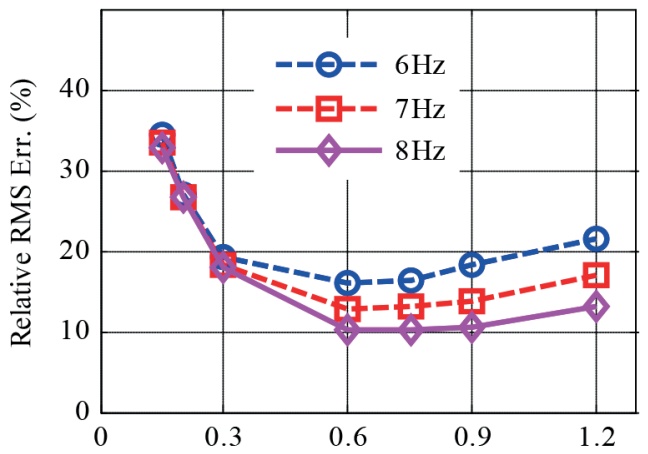

(c)

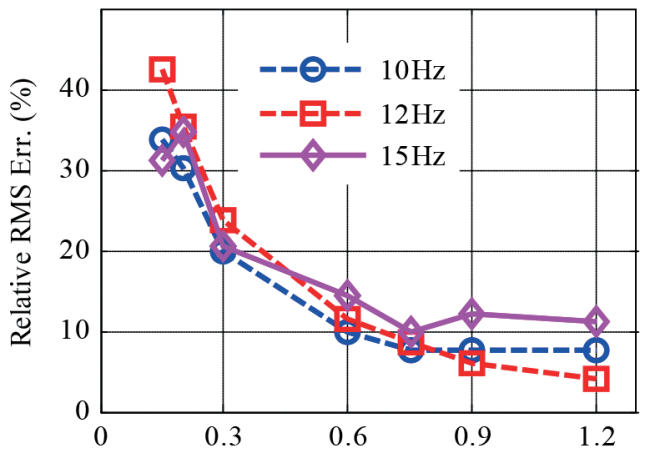

(d)

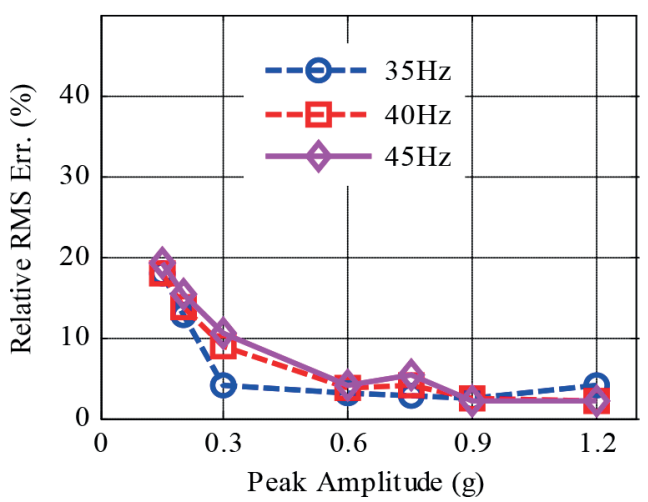

Fig. 6 Relative RMS error versus peak amplitude curves of uncompensated harmonic tests at (a) 2-, 4-, 5-; (b) 6-, 7-, 8-; (c) 10-, 12-, 15- and (d) 35-, 40-, $45-\mathrm{Hz}$, frequencies 
(a)

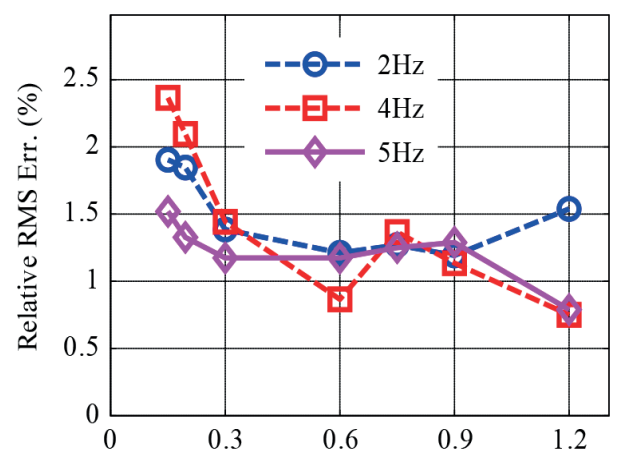

(b)

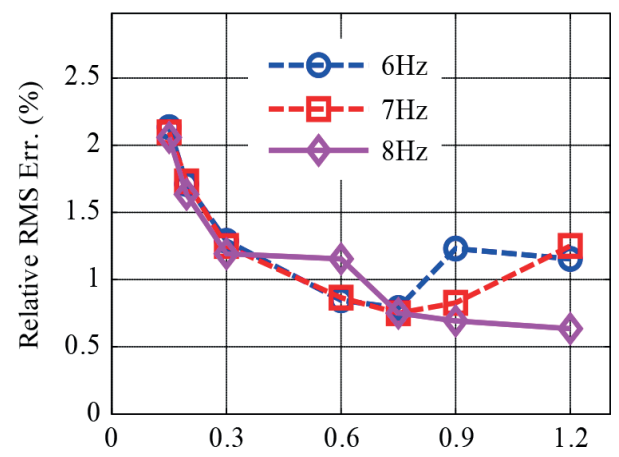

(c)

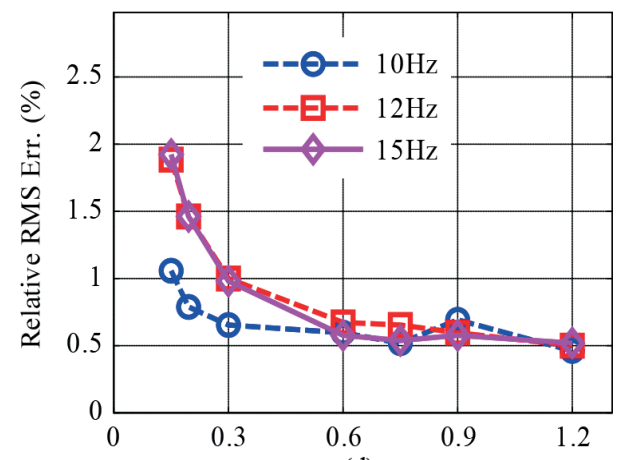

(d)

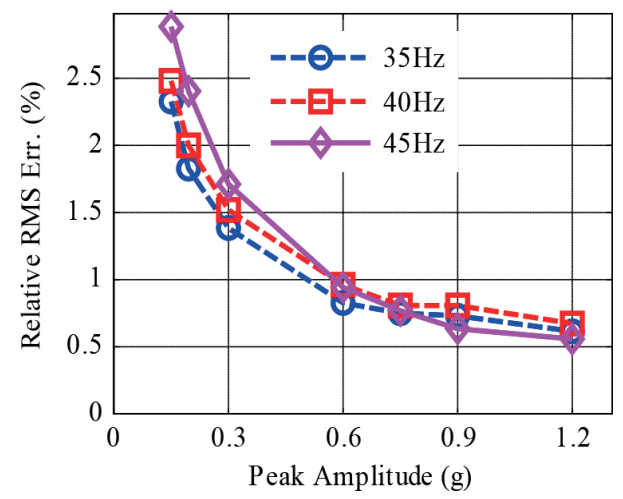

Fig. 7 Relative RMS error versus peak amplitude curves of compensated harmonic tests at (a) 2-, 4-, 5-; (b) 6-, 7-, 8-; (c) 10-, 12-, 15- and (d) 35-, 40-, $45-\mathrm{Hz}$, frequencies

The results of the relative RMS error of the four groups, obtained for the case of compensated tests, are shown through Fig. 7(a) to 7(d). As it can be seen in all graphs, the relative RMS error was significantly reduced, especially for peak amplitudes exceeding $0.3 \mathrm{~g}$ for which we obtained values of error less than $1.5 \%$. Therefore it is obvious how APC and AHC help to achieve a better system performance in terms of signal fidelity reproduction.

\subsection{Total Harmonic Distortion Comparison}

Total harmonic distortion (THD) is another important criterion by which we can measure the level of distortion of the shaking table response to harmonic command signal. It is defined by:

$$
T H D=\frac{\sqrt{A_{2}^{2}+A_{3}^{2}+A_{4}^{2}+\ldots}}{A_{1}} \times 100
$$

Where $\mathrm{A} 1$ is the fundamental amplitude, $\mathrm{A} 2$ is the amplitude of second harmonic; A3 is the amplitude of the third harmonic, and so on.

In Fig. 8, the results of the THD obtained for the case of uncompensated tests is shown. The figure shows strong harmonic distortion values (30 to $35 \%$ ) for tests with frequencies around $1 / 3$ of oil column frequency and low excitation amplitudes. In fact, spurious harmonics appeared in the table acceleration feedback signal and oil column frequency was excited. These unwanted harmonic components are mainly due to the nonlinearities on the servovalve. Furthermore, it can be seen from the figure that the THD tends to smaller values $(<5 \%)$ as the excitation frequency increases. Finally, it should be noted that for a given excitation frequency, the increase in the excitation amplitude tends to reduce the harmonic distortion of the table acceleration response, except for the frequency of $2 \mathrm{~Hz}$ for which we obtain an increase of the value of total harmonic distortion as the amplitude of excitation increases, due to the growing hydraulic demand and flow limits.

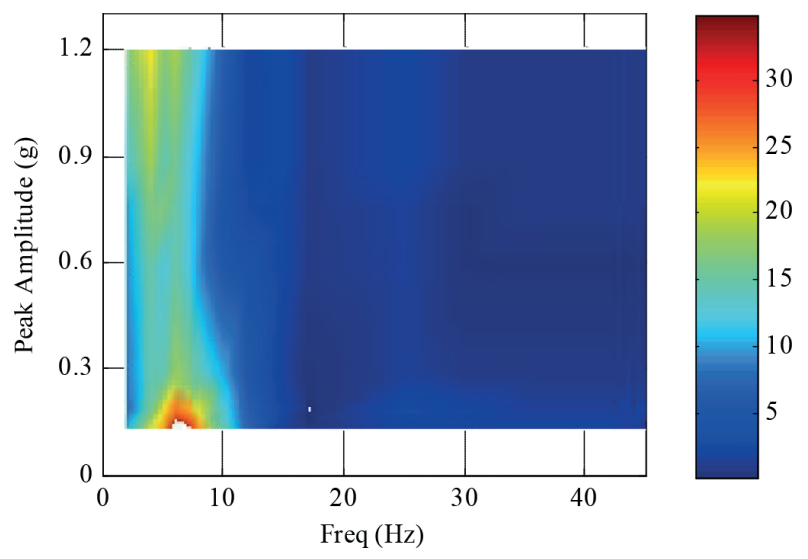

Fig. 8 Total harmonic distortion of uncompensated set

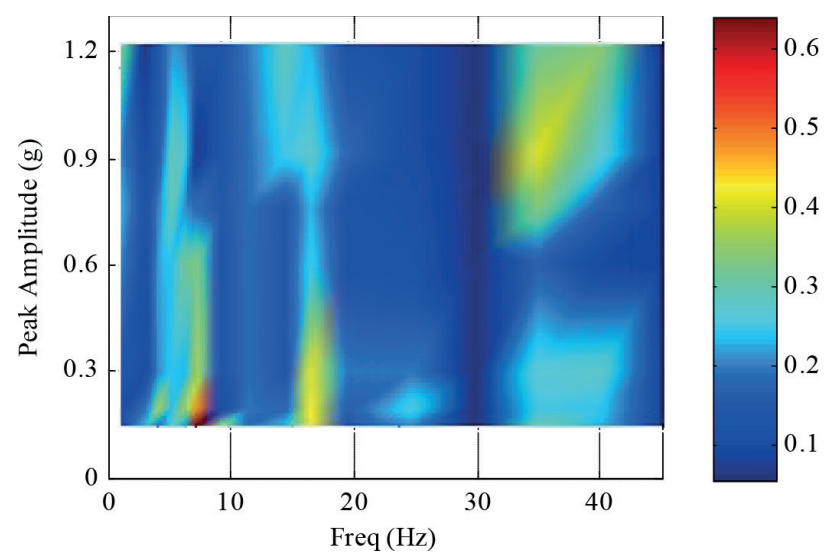

Fig. 9 Total harmonic distortion of compensated tests set 
The results of the THD obtained for the case of compensated tests are shown in Fig. 9. It can be seen clearly that the THD is very small $(<0.6 \%)$, regardless of the frequency and amplitude excitation. This demonstrates the effectiveness of these adaptive control techniques, mainly $\mathrm{AHC}$, in compensating the response of the shaking table.

\section{Cross coupling}

Geometric cross coupling between degrees of freedom occurs whenever actuators in one axis must react to loads due to motions in another axis [18]. In six degree of freedom shaking table systems, this effect arises regardless of which table axis is excited. For example, a table loaded with a specimen having a relatively high center of gravity above the shaking table platform, when excited in the horizontal direction, generates an Over Turning Moment (OTM) which produces rocking motion in pitch or roll.

Control algorithms using feed forward compensation can help to reduce, without removing completely, these undesirable cross talk effects. By using adaptive control techniques significant improvements of the motion performance of the table can be achieved.

In this section, the performances of $\mathrm{APC}$ and $\mathrm{AHC}$ on the reduction of dynamic cross-coupling of the CGS shaking were explored. For this purpose, series of harmonic tests were conducted on the table loaded with a resonant specimen. Detail of the tests are reported in table 1

Table 1 Tests performed for cross coupling investigation

\begin{tabular}{lccc}
\hline Test & Freq $(\mathrm{Hz})$ & Amplitude $(\mathrm{g})$ & APC+AHC \\
\hline S1 & 20 & 0.85 & Off (NC) \\
S2 & 20 & 0.85 & On (C) \\
S3 & 10 & 0.05 & Off (NC) \\
S4 & 10 & 0.10 & Off (NC) \\
S5 & 10 & 0.15 & Off (NC) \\
S6 & 10 & 0.05 & On (C) \\
S7 & 10 & 0.10 & On (C) \\
S8 & 10 & 0.15 & On (C)
\end{tabular}

\subsection{Test specimen}

Fig. 10 shows the specimen mounted on the CGS shaking table, used in tests performed for cross coupling investigation. The structure is a single-story braced steel frame, supporting four concrete blocks of a total mass of 20 tons. Additional damping is provided using two viscous dampers, type ALGAFD100, installed in the diagonals of the transversal direction which is the excitation axis. The design requirement was for a test specimen having a fundamental frequency of $10 \mathrm{~Hz}$, with sufficient height to cause significant overturning moment that will force the shaking table to undergo rolling.
The structure consists of IPE270 steel beams and HEA280 steel columns. The lateral mounting diagonals for fixing the dampers and the longitudinal X braces consist of UPN160 and UPN 80 sections, respectively. The specimen's plan dimensions are $2.50 \mathrm{~m} \times 2.00 \mathrm{~m}$. The centre of gravity is about $2.60 \mathrm{~m}$ above the shaking table platform. Three Setra ${ }^{\circledR}$-Model 141A accelerometers were fixed on the top of the specimen to measure its longitudinal, lateral and vertical acceleration responses.

The experimental identification of the test structure was performed [20], and the extracted natural frequencies and damping ratios are reported in table 2 .

Table 2 Specimen's natural frequencies and damping ratios

\begin{tabular}{lcc}
\hline Frequency $(\mathrm{Hz})$ & Damping ratio $(\%)$ & Direction \\
\hline 10.50 & 7.23 & Lateral \\
12 & 6.12 & Longitudinal \\
30 & 1.80 & Vertical \\
\hline
\end{tabular}
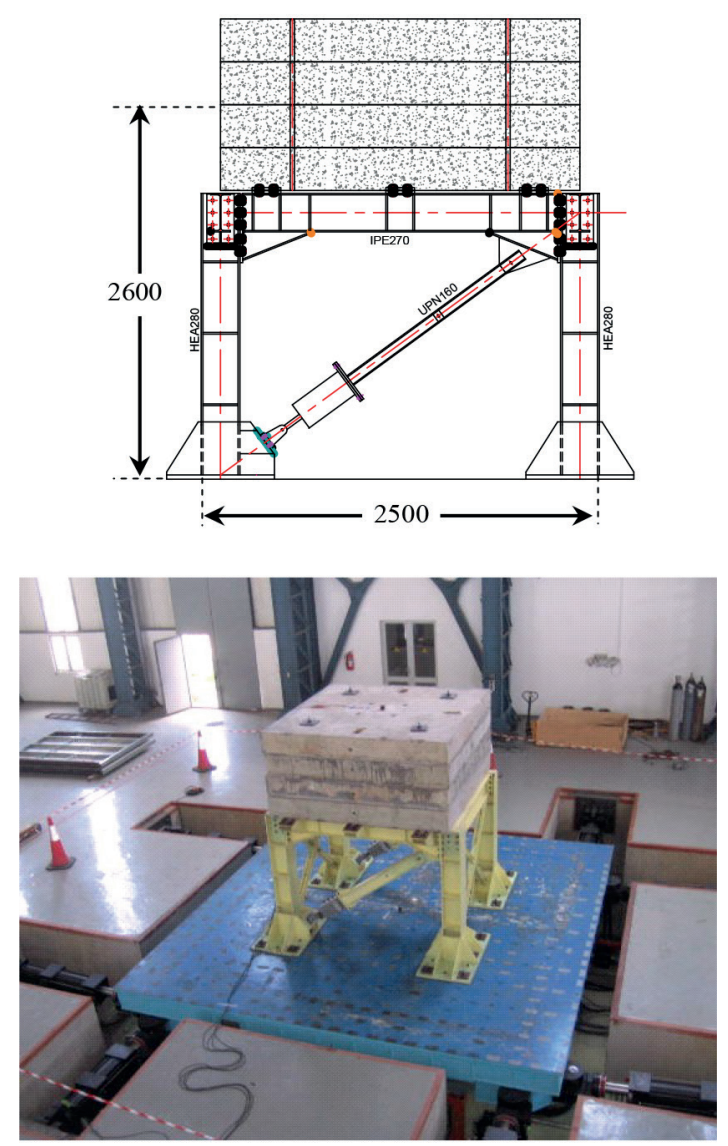

Fig. 10 View of the test specimen

\subsection{Results}

Fig. 11 shows the compensated (C) and uncompensated (NC) feedback acceleration plots, of the three translational axes of the CGS shaking table, due to the harmonic excitations S2 and S1, applied in the lateral axis. As it can be seen, very low spurious motions, in the longitudinal and the vertical axes are observed, as illustrated in Figs. 11(a, c). In fact, it was found that the peak values of the longitudinal spurious motions with respect to the corresponding transversal axis are less than 
$7.34 \%$ for the case of the uncompensated test S1 and 4.58\% for the compensated test $\mathrm{S} 2$. The corresponding values for the vertical axis are $5.41 \%$ and $3.84 \%$, respectively. These typical results reveal that cross-coupling between transverse and longitudinal directions in one hand and between transverse and vertical directions in other hand are negligible.

Fig. 12 shows the compensated (C) and uncompensated (NC) rotational acceleration feedback plots, of the CGS shaking table, due to tests $\mathrm{S} 2$ and S1, applied in the lateral axis. As observed, the uncompensated test $\mathrm{S} 1$ highlights a strong crosscoupling between transversal and roll axes, see Fig. 12(a), and a relatively low cross-coupling between the transversal and the two other rational axes, Figs. 12(b, c). Indeed, peak values of $42 \mathrm{deg} / \mathrm{s}^{2}, 7.60 \mathrm{deg} / \mathrm{s}^{2}$ and $11.2 \mathrm{deg} / \mathrm{s}^{2}$ are obtained for the roll, pitch and yaw motions, respectively.

In contrast, the efficiency of $\mathrm{APC}$ and $\mathrm{AHC}$ on the reduction of the cross-coupling effects is reflected on the graphs of the compensated test S2 (shown by doted lines in Figs. 12(a-c)). It is clearly shown that the spurious motions of the rotational axes are significantly reduced, particularly the roll motion in which the adaptive controls (APC and AHC) produce an approximately $85 \%$ reduction in peak value.

(a)
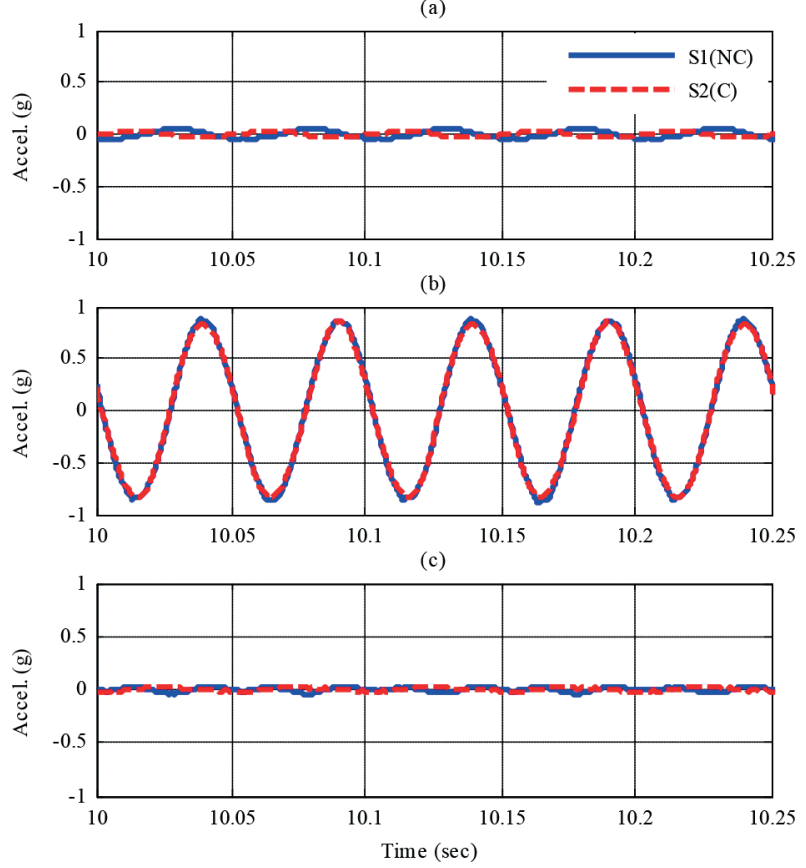

Fig. 11 Translational accelerations achieved on the CGS shaking table, (a): Longitudinal, (b): Tansversal and (c): Vertical, for sinusoidal tests S1 and S2
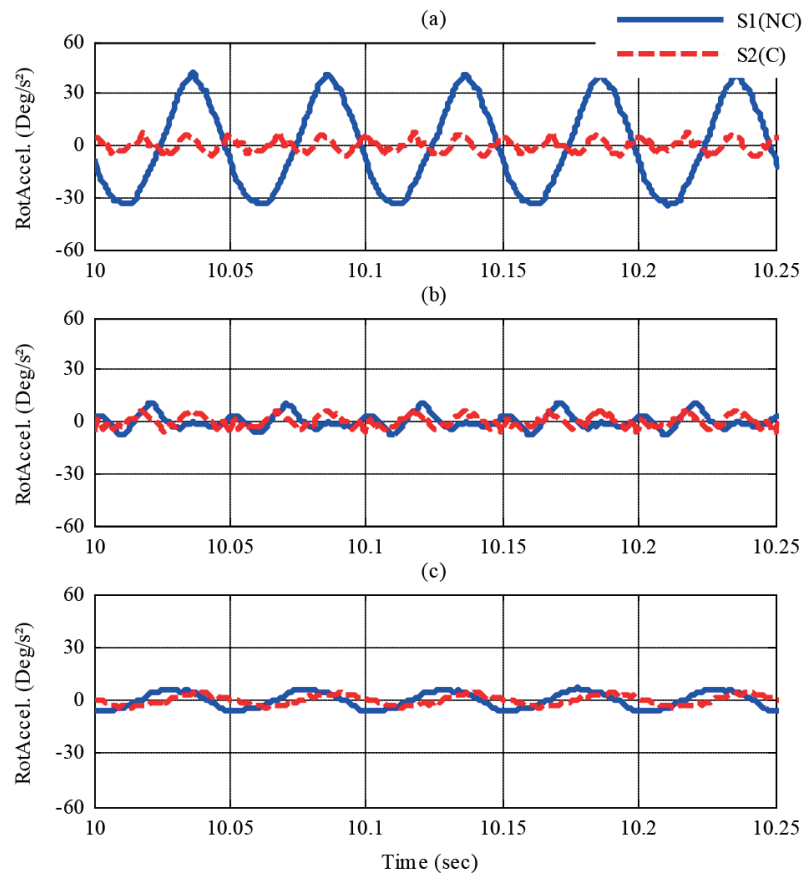

Fig. 12 Rotational accelerations achieved on the CGS shaking table, (a):Roll, (b): Pitch and (c): Yaw, for sinusoidal tests S1 and S2

Fig. 13 and Fig. 14, respectively show, the effects of varying the peak amplitude of the transversal harmonic excitations on the roll accelerations feedbacks of the CGS shaking table, for both cases uncompensated tests (S3, S4 and S5) and compensated tests (S6, S7 and S8). As expected, it is apparent from Fig. 13 that increasing the peak amplitude of the transversal harmonic excitation input leads to more important rotational motion in the roll axis and thus, to stronger cross-coupling between transversal and roll axes. Fig. 14, however, shows a noticeably lower roll motions, peak value, less than $2.5 \mathrm{deg} / \mathrm{s}^{2}$, are obtained regardless of the value of the peak amplitude of excitation inputs. Therefore, it is worth to mention that APC and AHC enable a significant reduction of the cross-coupling effects.

Finally for completeness, Fig. 15 shows a plot for $45 \mathrm{sec}$ of the compensated tests (S6, S7 and S8), where effect of APC and AHC in action is highlighted. As it can be seen, during the tests, the roll acceleration feedback continues to be reduced until it converges to its minimum possible value. However, it is clearly shown in the figure that the time of convergence increases as the amplitude of the test increases. This may be a major issue particularly in the case of short duration tests with high amplitudes. 


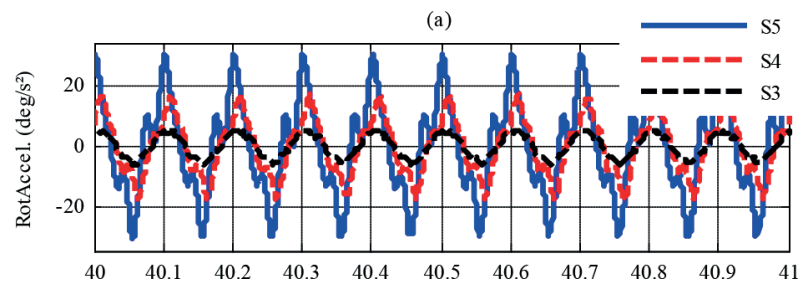

Fig. 13 Roll acceleration feedbacks of the CGS shaking for sinusoidal tests $\mathrm{S} 3, \mathrm{~S} 4$ and $\mathrm{S} 5$

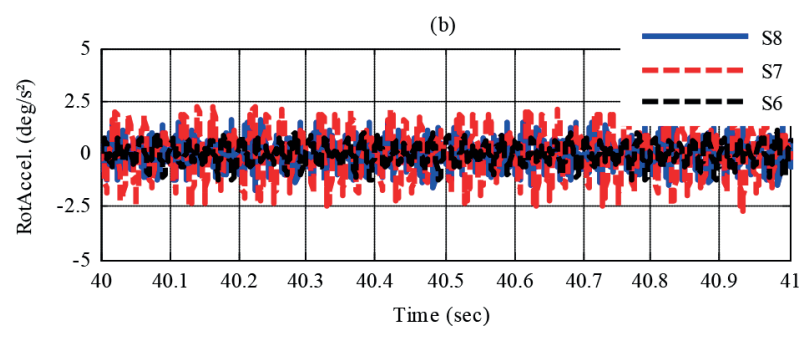

Fig. 14 Roll acceleration feedbacks of the CGS shaking for sinusoidal tests $\mathrm{S} 6, \mathrm{~S} 7$ and $\mathrm{S} 8$

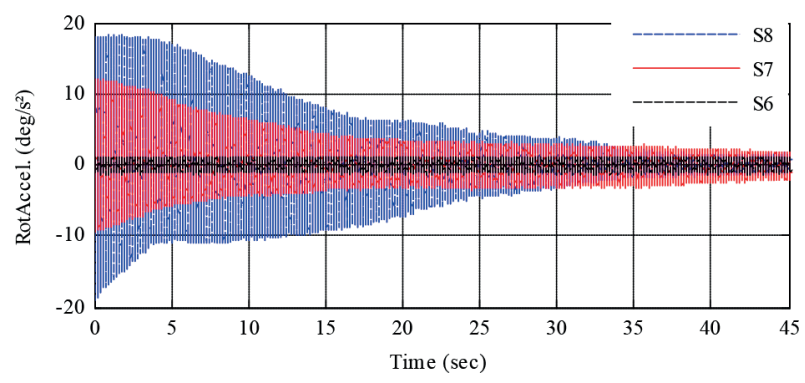

Fig. 15 Roll acceleration feedbacks of the CGS shaking for sinusoidal tests $\mathrm{S} 6, \mathrm{~S} 7$ and $\mathrm{S} 8$

\section{Conclusions}

Faithful reproduction of the desired motion is a complex subject and remains the most challenging tasks within the shaking table testing. Various compensation control strategies, all aimed to tackle this critical issue, have been proposed. In this article, the results of the experimental investigation on harmonic signal tracking performances of the CGS shaking table were presented.

The test program was divided into two main phases. The first phase consists on the bare table testing condition. A set of uncompensated tests was conducted and lack of fidelity in harmonic signal reproduction was quantified in term of relative RMS error and total harmonic distortion (THD), for each test. It was found that parameters like servovalve characteristics and oil column resonant frequency constitute the most predominant factors that can affect the shaking table performance. Indeed, the experimental program revealed, that high signal distortion occurs for signals with frequencies lower than $1 / 3$ of the oil column $(2-5 \mathrm{~Hz})$, which is due to the nonlinearities in the shaking table. The distortion becomes more pronounced as the acceleration amplitude increases. In contrast, amplitude attenuation and phase delay were observed for signals with moderate and high frequencies.
Subsequently, a second set of compensated tests using Amplitude Phase Control (APC) and Adaptive Harmonic Cancellation (AHC), which are developed for a particular case of cyclic waveform tests, were performed in order to assess their effectiveness. A maximum value of relative RMS error of $2.5 \%$ and a THD less than $0.6 \%$ were obtained for all tests. It was then, demonstrated that perfect harmonic acceleration signal reproduction was achieved while using APC and AHC whatever was the acceleration and the frequency levels. As an example, the RMS error was less than $1.5 \%$ for peak amplitude excitation exceeding $0.3 \mathrm{~g}$.

In the second phase of testing the cross-coupling effects and the ability of APC and AHC on their reduction were explored. To this end, compensated and uncompensated harmonic tests have been conducted on the shaking table loaded with a 20tons specimen of $10 \mathrm{~Hz}$. By analyzing the tests results, it was found that the control system was unable to eliminate the clearly high cross-coupling effects, mainly between transversal and roll axis, for the case of uncompensated test. Peak values of 42 $\mathrm{deg} / \mathrm{s}^{2}, 7.60 \mathrm{deg} / \mathrm{s}^{2}$ and $11.2 \mathrm{deg} / \mathrm{s}^{2}$ were obtained for the roll, pitch and yaw motions, respectively. In contrast, theses effects were significantly reduced by $85 \%$ in the case of the compensated tests in which APC and AHC were used. As an example, it was shown that noticeable low roll motions, peak value, less than $2.5 \mathrm{deg} / \mathrm{s}^{2}$, are obtained regardless of the value of the peak amplitude of excitation inputs.

As a conclusion and based on the experimental tests results, it can be concluded that APC and AHC represent very powerful and attractive techniques which greatly improve the fidelity of the shaking table system's response to a harmonic wave command signal. Moreover, it should be mentioned that the success of their use is highly dependent on the skills and experience of the operator. Also, great caution should be taken especially when using AHC in order to prevent instability that could destroy test-specimen.

\section{Reference}

[1] Severn, R. T. "The development of shaking tables-A historical note". Earthquake Engineering \& Structural Dynamics, 40(2), (2011), pp. 195213. 2010. https://doi.org/10.1002/eqe.1015

[2] Stehman, M., Nakata, N. "Direct acceleration feedback control of shake tables with force stabilization". Journal of Earthquake Engineering, 17(5), pp. 736-749. 2013. https://doi.org/10.1080/13632469.2013.776508

[3] Clark, A., J., Cross, D. J. “The effect of specimen resonances on accurate control of multiple degree-of-freedom servohydraulic shaking tables". In: $8^{\text {th }}$ World Congress on Earthquake Engineering. San Francisco, California, U.S.A 1984. 6, pp. 47-54. http://www.iitk.ac.in/nicee/wcee/article/8_vol6_47.pdf

[4] Conte, J. P., Trombetti, T. L. "Linear dynamic modeling of a uni-axial servohydraulic shaking table system”. Earthquake Engineering \& Structural Dynamics, 29(9), pp. 1375-1404. 2000. https://doi.org/10.1002/10969845(200009)29:9<1375::AID-EQE975>3.0.CO;2-3 
[5] Kuehn. J. D., Epp, D., Patten, W. N. "High-fidelity control of a seismic shake table". Earthquake Engineering \& Structural Dynamics, 28(11), pp. 1235-1254. 1999. https://doi.org/10.1002/(SICI)1096-9845 (199911)28:11<1235::AID-EQE864>3.0.CO;2-H

[6] Luco, J. E., Ozcelik, O., Conte, J. P. "Acceleration Tracking Performance of the UCSD-NEES Shake Table". Journal of Structural Engineering, 136(5), pp. 481-490. 2010. https://doi.org/10.1061/(ASCE) ST.1943-541X.0000137

[7] Tagawa, Y., Kajiwara, K. "Controller development for the E-Defense shaking table". In: Proceedings of the Institution of Mechanical Engineers, Part I: Journal of Systems and Control Engineering, 221(2), pp. 171-181. 2007. https://doi.org/10.1243/09596518JSCE331

[8] Chen, C., Ricles, J. M, "Large-scale real-time hybrid simulation involving multiple experimental substructures and adaptive actuator delay compensation”. Earthquake Engineering \& Structural Dynamics, 41(3), pp. 549-569. 2012. https://doi.org/10.1002/eqe.1144

[9] Shen Gang, Zhu Zhen-Cai, Zhang Lei, Tang Yu, Yang Chi-fu, Zhao Jinsong, Liu Guang-da, Han Jun-Wei "Adaptive feed-forward compensation for hybrid control with acceleration time waveform replication on electro-hydraulic shaking table". Control Engineering Practice, 21(8), pp. 1128-1142, 2013. https://doi.org/10.1016/j.conengprac.2013.03.007

[10] Gang Shen, Zhen-Cai Zhu, Xiang Li, Yu Tang, Dong-Dong Hou, WenXiang Teg. "Real-time electro-hydraulic hybrid system for structural testing subjected to vibration and force loading". Mechatronics, 33, pp. 49-70. 2016. https://doi.org/10.1016/j.mechatronics.2015.10.009

[11] Nakata, N. "A multi-purpose earthquake simulator and a flexible development platform for actuator controller design". Journal of Vibration and Control, 18(10), pp. 1552-1560. 2010. https://doi. org/10.1177/1077546311421946

[12] Stoten, D. P., Shimizu, N, "The feedforward minimal control synthesis algorithm and its application to the control of shaking-tables". In: Proceedings of the Institution of Mechanical Engineers, Part I, Journal of Systems and Control Engineering, 221(3), pp. 423-444. 2007. https://doi. org/10.1243/09596518JSCE246
[13] Chengwen, W., Zongxia, J., Shuai, W., Yaoxing, S. "Nonlinear adaptive torque control of electro-hydraulic load system with external active motion disturbance". Mechatronics, 24(1), pp. 32-40. 2014. https://doi. org/10.1016/j.mechatronics.2013.11.005

[14] Seki, K., Iwasaki, M., Kawafuku, M., Hirai, H., Yasuda, K, “Adaptive Compensation for Reaction Force With Frequency Variation in Shaking Table Systems". In: IEEE Transactions on Industrial Electronics, 56(10), pp. 3864-3871. 2009. https://doi.org/10.1109/TIE.2009.2022519

[15] Seki, K., Iwasaki, M. "Disturbance suppression control combined disturbance observer with acceleration feedback in 2-dimensional shaking table system". In: Advanced Intelligent Mechatronics (AIM), 2014 IEEE/ ASME International Conference. Besacon, 2014, pp. 156-161. https:// doi.org/10.1109/AIM.2014.6878071

[16] Thoen, B. K. 469D Seismic Digital Control Software, MTS Systems Corporation. 2004.

[17] Airouche, A., Bechtoula, H., Aknouche, H., Thoen, B. K., Benouar, D. "Experimental identification of the six DOF CGS, Algeria shaking table system". Smart Structures and Systems, 13(1), pp. 137-154. 2014. http:// dx.doi.org/10.12989/sss.2014.13.1.137

[18] Kusner, D. A., Rood, J. D., Burton, G. W. "Signal reproduction fidelity of servohydraulic testing equipment". In: $10^{\text {th }}$ World Congress on Earthquake Engineering. Rotterdam, The Netherlands. pp. 2683-2688. 1992.

[19] Clark, A. "Dynamic Characteristics of Large Multiple Degrees Of Freedom Shaking Tables". In: $10^{\text {th }}$ World Congress on Earthquake Engineering. Rotterdam, The Netherlands. pp. 2823-2828. 1992.

[20] Aknouche, H.; Bechtoula, H.; Airouche, A., Benouar, D. "Investigation on the performance of the six DOF CGS, Algeria, shaking table". Earthquakes and Structures, 6(5), pp. 539-60. 2014. http://dx.doi. org/10.12989/eas.2014.6.5.539 